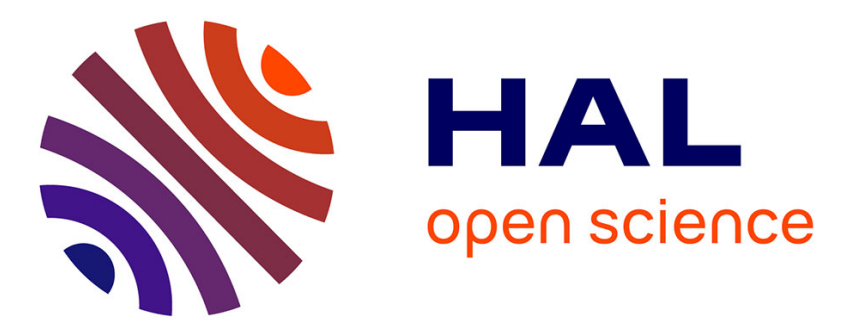

\title{
Light-induced waveguide by a finite self-trapped vortex beam in a photorefractive medium
}

R. Passier, M. Chauvet, B. Wacogne, F. Devaux

\section{To cite this version:}

R. Passier, M. Chauvet, B. Wacogne, F. Devaux. Light-induced waveguide by a finite self-trapped vortex beam in a photorefractive medium. Journal of Optics, 2011, 13, pp.085502. 10.1088/20408978/13/8/085502 . hal-00655050

\section{HAL Id: hal-00655050 \\ https://hal.science/hal-00655050}

Submitted on 6 May 2021

HAL is a multi-disciplinary open access archive for the deposit and dissemination of scientific research documents, whether they are published or not. The documents may come from teaching and research institutions in France or abroad, or from public or private research centers.
L'archive ouverte pluridisciplinaire HAL, est destinée au dépôt et à la diffusion de documents scientifiques de niveau recherche, publiés ou non, émanant des établissements d'enseignement et de recherche français ou étrangers, des laboratoires publics ou privés. 


\title{
Light-induced waveguide by a finite self- trapped vortex beam in a photorefractive medium
}

\author{
Rémy Passier, Mathieu Chauvet, Bruno Wacogne \\ and Fabrice Devaux
}

Département d'Optique P.M. Duffieux, Institut FEMTO-ST, UMR CNRS 6174, Université de

Franche-Comté, 16 route de Gray, F-25030 Besançon Cedex, France

\begin{abstract}
We report the formation of a $2 \mathrm{D}$ waveguide induced by a self-trapped vortex beam in a photorefractive-photovoltaic medium. Demonstrations are performed by monitoring the self-trapping dynamic of an annular vortex beam in a $\mathrm{LiNbO}_{3}: \mathrm{Fe}$ crystal. Control of the experimental observations is consistent with the underlying physics revealed by a numerical model.
\end{abstract}

Keywords: electro-optical effects, photorefractive materials, photorefractive optics, self-focusing, optical waveguides

\section{Introduction}

During the last two decades, many techniques have been developed to achieve optically induced 3D light circuits, including micromachining [1] and optical masks [2, 3] as well as soliton-induced waveguides [4]. In particular, optical spatial solitons have been at the center of numerous studies to demonstrate fundamental properties [5] and to induce low loss reconfigurable waveguides [6]. The versatility of solitons has been shown using different optical nonlinearities such as the Kerr [7] or quadratic effect [8] and in different media like glasses [9], semi-conductors [10], or liquid crystals [11]. Solitons in photorefractive medium have been particularly studied since they can be formed at very low light power due to the high sensitivity of these materials. The first theoretical [12] and experimental [13] photorefractive solitons were produced using a static applied electric field. These photorefractive (PR) solitons are formed by the creation of an internal space charge field that modifies the refractive index by the Pockels effect. This effect can lead to the formation of bright [14] or dark PR solitons [15], depending on the sign of the applied electric field. The models [16-19] are now mature and experimental realization of $(2+1)$-D solitons has led to the study of more complex configurations such as the formation of junctions [20, 21]. Recently, Fazio et al [22] demonstrated beam self-focusing in a widely used photonic medium, $\mathrm{LiNbO}_{3}$, by using a high applied voltage. To avoid the application of high electric field an alternative way consists of using the photovoltaic field. One-dimensional dark [23] or bright [24] solitons, can be realized in a defocusing or a focusing medium, respectively.

Since $\mathrm{LiNbO}_{3}$ is intrinsically a defocusing medium because of the photovoltaic effect, trapping of a vortex beam is a natural solution to form two-dimensional guiding structures. These beams are already used in different fields of physics for the manipulation of molecules by optical tweezers [25] or to study Bose-Einstein condensates [26]. However, a vortex beam has an extended light distribution that is prone to dislocations in the nonlinear regime because of modulation instability [27, 28]. Two-dimensional dark vortex solitons have been obtained using an applied field in a photorefractive SBN crystal [29], or by using the photovoltaic effect in a $\mathrm{LiNbO}_{3}: \mathrm{Fe}$ crystal [30], creating the vortex beam by the help of a helicoidal phase.

Practically, vortex beams of small size such as smallring intensity profile beams could be useful to induce multiple waveguides close to each other in a sequential manner with no overlap. Moreover, such vortex beams are easy to form. The behavior of small size vortex beams propagating in a nonlinear photorefractive medium consequently deserves a 

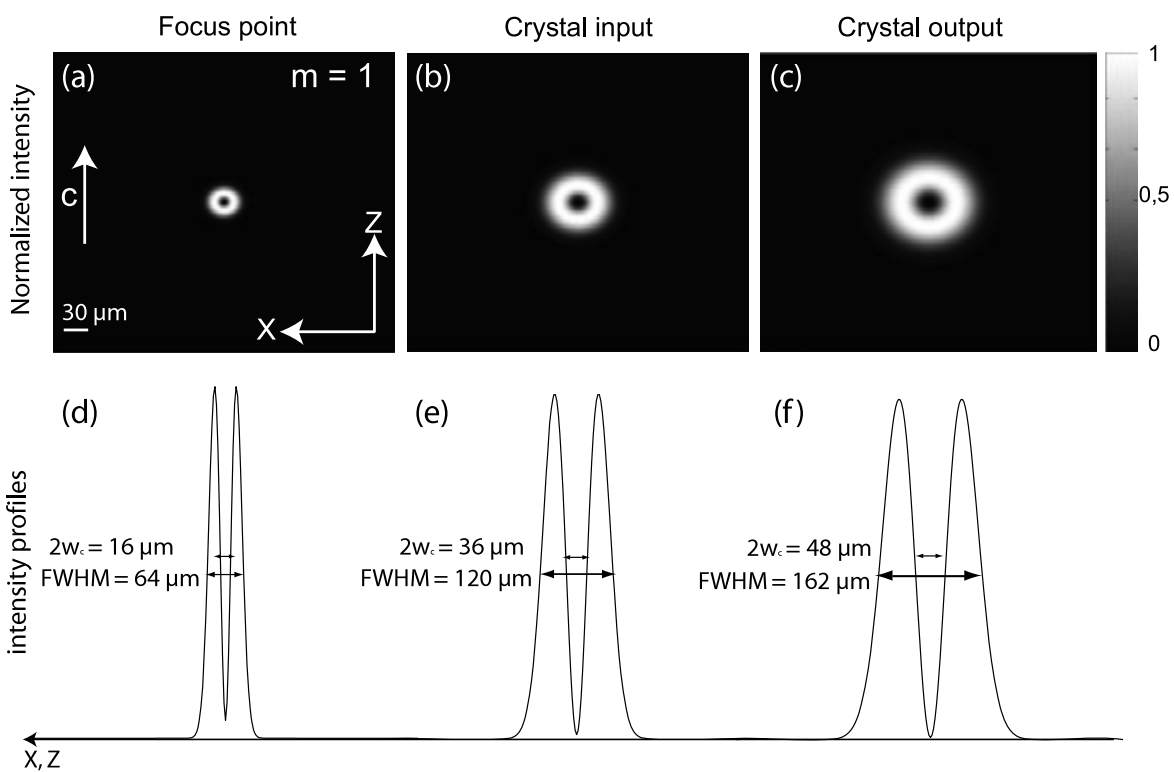

Figure 1. Calculated intensity distributions in the linear regime at the focal point (a), at the input (b) and at the output (c) faces of a $9 \mathrm{~mm}$ long $\mathrm{LiNbO}_{3}: \mathrm{Fe}$ crystal. The respective intensity profiles along the $Z$ and $X$ axes ((d)-(f)) are also shown.

specific study to get a better insight, which is the aim of this paper. More specifically we will consider finite size optical vortices to create self-trapped beams. $(2+1)$-D self-trapped beams in a defocusing photovoltaic media will be tackled both numerically and experimentally. Additionally, the potential to write waveguides inside bulk $\mathrm{LiNbO}_{3}$ is assessed.

\section{Numerical analysis}

To determine the optimum conditions to obtain a soliton-like propagating beam that would induce automatically a singlemode waveguide, a numerical model has been developed [31]. This model allows calculation of the space charge field in 3D, based on a one carrier and one deep center model, and is derived from $[12,32]$

$$
\begin{gathered}
\frac{\partial N_{\mathrm{D}}^{+}}{\partial t}=s\left(I+I_{\mathrm{d}}\right)\left(N_{\mathrm{D}}-N_{\mathrm{D}}^{+}\right)-\gamma N_{\mathrm{e}} N_{\mathrm{D}}^{+} \\
\vec{\nabla} \cdot\{[\varepsilon] \vec{E}\}=\rho \\
\rho=\mathrm{e}\left(N_{\mathrm{D}}^{+}-N_{\mathrm{A}}-N_{\mathrm{e}}\right) \\
\vec{J}=\mathrm{e} \mu N_{\mathrm{e}} \vec{E}+\mu k_{\mathrm{B}} T \vec{\nabla} N_{\mathrm{e}}+\beta_{\mathrm{ph}}\left(N_{\mathrm{D}}-N_{\mathrm{D}}^{+}\right) I \vec{c} \\
\frac{\partial \rho}{\partial t}=-\vec{\nabla} \cdot \vec{J}
\end{gathered}
$$

where $I_{\mathrm{d}}$ is the equivalent dark intensity, $N_{\mathrm{A}}, N_{\mathrm{D}}, N_{\mathrm{D}}^{+}$and $N_{\mathrm{e}}$ are respectively the densities of shallow acceptors, deep donors, deep ionized donors and free electrons. $s$ is the photoexcitation coefficient, $\gamma$ is the recombination constant, $k_{\mathrm{B}}$ is the Boltzmann constant, $\mu$ is the electron mobility and $T$ is the temperature. The photovoltaic current directed along the $\mathrm{LiNbO}_{3} c$-axis (i.e. the $Z$ axis) is considered throughout $\beta_{\mathrm{ph}}$ which is a light polarization dependent component of the photogalvanic tensor. $\beta_{13}$ is used for ordinary polarized beams. $[\varepsilon]$ is the static dielectric tensor, $\rho$ is the charge density and $\vec{J}$ is the current density. In addition, the dynamic of the photorefractive effect is resolved and the intrinsic anisotropy of the photorefractive effect is taken into account [33]. The small size ring vortex beam is given by [34]

$$
\overrightarrow{U_{e m}}(\vec{r})=\vec{A}\left(r_{\perp}, z\right) \exp (-\mathrm{i}(\omega t-k z)) \exp (\mathrm{i} m \theta)
$$

where $r_{\perp}$ represents the coordinates in the transverse plane and $z$ is the position along the propagation axis, $m$ is the topological charge of the vortex, $\theta$ is the azimuth angle and $\vec{A}=\overrightarrow{A_{0}}\left(r_{\perp} / \omega_{0}\right)^{|m|} \exp \left(-r_{\perp}^{2} / 2 \omega_{0}^{2}\right)$ is the optical field amplitude.

This model is then used to resolve the usual following light wave equation, linking the optical field amplitude $\vec{A}$ to the index modulation $\Delta n$ :

$$
\frac{\partial}{\partial z} \vec{A}\left(r_{\perp}, z\right)=\mathrm{i}\left(\frac{1}{2 k} \frac{\partial^{2}}{\partial r_{\perp}^{2}}+\frac{2 \pi}{\lambda} \Delta n\right) \vec{A}\left(r_{\perp}, z\right) .
$$

To describe the experimental configuration further we consider the propagation of a vortex beam at a wavelength of $473 \mathrm{~nm}$ focused to a $25 \mu \mathrm{m}$ waist. This one-charged vortex $(m=1)$ is represented in figure 1 . The beam waist is positioned before the crystal, at a distance equal to a beam Rayleigh distance of $6.8 \mathrm{~mm}$. It has an external beam size diameter (or FWHM, full width at half maximum) of $120 \mu \mathrm{m}$ and a dark core diameter of $36 \mu \mathrm{m}$ at the crystal input (figures 1(b) and (e)). After $9 \mathrm{~mm}$ propagation in the linear regime in the iron doped $\mathrm{LiNbO}_{3}$ the beam diffracts at the output to $162 \mu \mathrm{m}$ for the external diameter and $48 \mu \mathrm{m}$ for the dark core (figures 1(c) and (f)).

In a second set of numerical calculation we assume that the medium possesses photorefractive properties dictated by the photovoltaic effect. The self-trapping dynamic during the transient regime of the vortex beam is then monitored and 

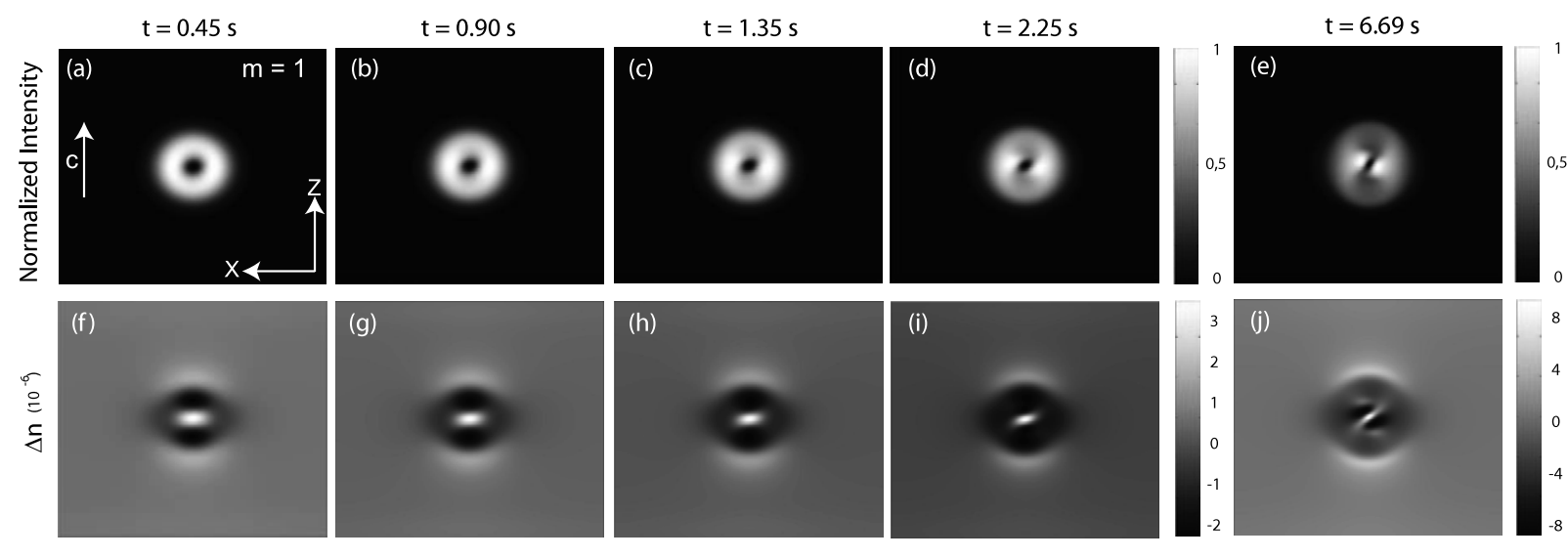

Figure 2. Temporal evolution of a one-charged vortex $(m=1)$ at the exit face with the same parameters as in figure 1 but in the nonlinear regime at different instants of the induction process ((a)-(e)). The respective refractive index distributions are also presented ((f)-(j)).

presented in figure 2. Propagation along the $Y$ axis of the $\mathrm{LiNbO}_{3}: \mathrm{Fe}$ crystal is chosen to avoid anisotropy playing an active role in the process [33]. Beam profiles are shown along $X$ and $Z$. For a close fit with the values given in the literature and the physical experiment described further, the following parameters are set: $I_{\mathrm{d}}=1 \times 10^{4} \mathrm{~W} \mathrm{~m}^{-2}, E_{\mathrm{ph}}=$ $-7,7 \times 10^{6} \mathrm{~V} \mathrm{~m}^{-1}, N_{\mathrm{A}}=5 \times 10^{22} \mathrm{~m}^{-3}$ and $N_{\mathrm{D}} / N_{\mathrm{A}}=1.1$. A peak power density of $I_{\max } \simeq 3 \times 10^{6} \mathrm{~W} \mathrm{~m}^{-2}$ which gives a ratio $I_{\max } / I_{\mathrm{d}} \simeq 263$ is used. Other parameters are taken from [35]. Since an unintentionally doped medium is used it is fair to consider a low concentration of deep donor around $10^{19} \mathrm{~m}^{-3}$ [36].

Figures 2(a)-(e) show the temporal intensity distribution as the nonlinear effect is taking place. In addition, the corresponding refractive index distribution dynamic written in the material is represented (figures $2(\mathrm{f})-(\mathrm{j})$ ). The last two figures (figures 2(e)-(j)) depict a strongly distorted beam. The beam intensity distribution is measured at the output of the crystal while the nonlinear effect takes place in the photovoltaic-photorefractive material. We observe that the nonlinear effect tends to elongate the beam more strongly along the $Z$ axis which corresponds to the direction of the defocusing photovoltaic field $(\vec{c})$ (figure 2(b)). During this transient regime brighter areas appear on the left and right sides of the beam (figures 2(b)-(d)). Such a beam distortion is mainly due to the presence of low index areas on the bottom and top parts of the beam. Note that away from the illuminated areas, the refractive index change is due to the space charge field formed by the photovoltaic current loops present in this open-circuit configuration. For instance, an index increase is observed above and below the illuminated region while a slight index decrease is present on the right and left sides. Moreover symmetric low index lobes are present above and below the central core and form an elliptical high index area (figures 2(f)-(i)). This dynamic leads to the self-focusing of the dark core for which a stronger refractive index is present compared to the surrounding region, thus forming a potential $2 \mathrm{D}$ waveguide. These results confirm earlier published results $[18,33]$. After $t=1.35 \mathrm{~s}$, beam expansion and core focusing continue (figures 2(d) and (i)) and can lead to strong distortion (figures 2(e) and (j)) and even beam dislocation, showing that a steady-state spatial soliton cannot be reached. One way to produce proper waveguides is to stop the nonlinear phenomenon before appearance of this dislocation.

The detailed evolution of the vortex core and external diameters is analyzed to better visualize the dynamical behavior before beam distortion. Figure 3 represents both diameters along the $X$ and $Z$ axes. It clearly reveals the gradual increase of the external diameter while the internal core is shrinking. The anisotropy of the photorefractive effect has a strong influence on the evolution of the external beam diameter as shown by the appearance of a marked ellipticity (figure 3(b)). However the dark core looks circular (figure 3(a)) as the beam ellipticity is tilted by about $45^{\circ}$. After $t=1.35 \mathrm{~s}$ we observe that a similar core size of about $36 \mu \mathrm{m}$ is present at the input and output faces of the crystal. If the induction process is stopped at that moment, before excessive focussing and any dislocation process occur, a homogeneous waveguide is expected to be memorized across the crystal, written by this finite size vortex beam.

In the following, the induced waveguides are analyzed with a probe beam at $473 \mathrm{~nm}$ with an ordinary polarization. The asymmetric photo-induced index transverse distribution is depicted in figure 4(a). The index profiles along $X$ (figure 4(d)) and $Z$ (figure $4(\mathrm{~g})$ ) are particularly explicit in highlighting the asymmetry. The modulation depth is more than two times as much along the polar axis $Z\left(\Delta n=4 \times 10^{-6}\right)$ as it is in the perpendicular direction $\left(\Delta n=1.75 \times 10^{-6}\right)$. The low index area surrounding the higher refractive index center forms a guiding structure. This guiding structure is first probed by a Gaussian beam which is larger than the central guiding region (FWHM of $130 \mu \mathrm{m}$ ). This provides a way to visualize the overall index distribution written in the medium as shown in figure 4(b).

To evaluate the guiding properties of the central area, the probe beam is then focused at the entrance face to a spot similar in size to the vortex core. The guided beam reveals an elliptical beam profile at the crystal output as shown in figure 4(c). The mode of this single-mode waveguide is $61 \mu \mathrm{m}$ along the $X$ axis and $36 \mu \mathrm{m}$ along the $Z$ axis (figures 4(f) and (i)), with a deeper 

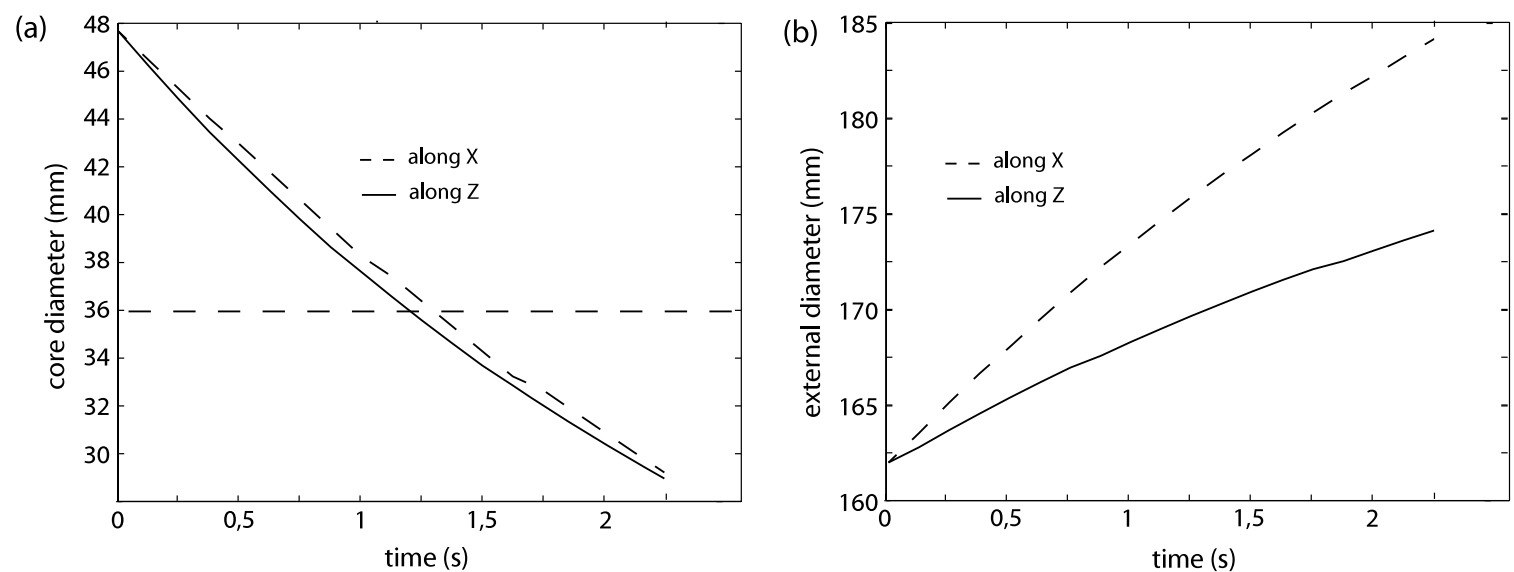

Figure 3. Temporal evolution of the core (a) and external (b) beam diameters (FMHM) for the numerical experiment corresponding to figure 2 from $t=0$ to $t=2.25 \mathrm{~s}$, along the $X$ (dashed line) and $Z$ axes. The horizontal dashed line indicates the core diameter at the input face of the photorefractive medium.
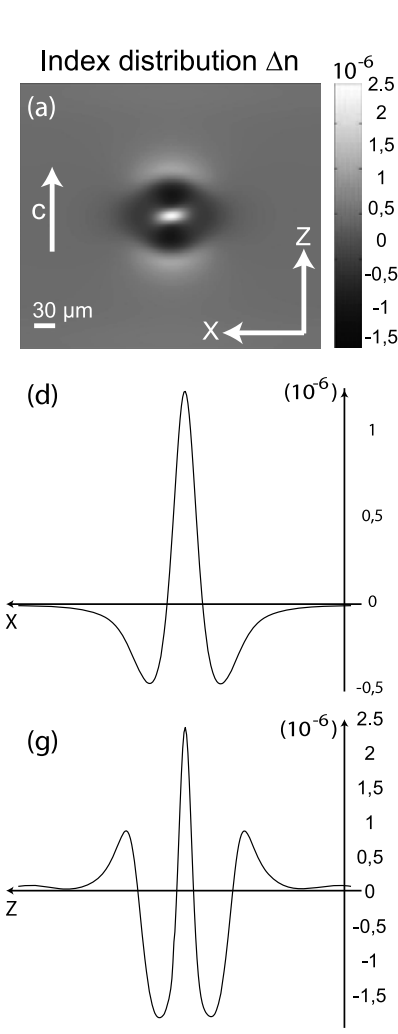

Intensity

with large probe

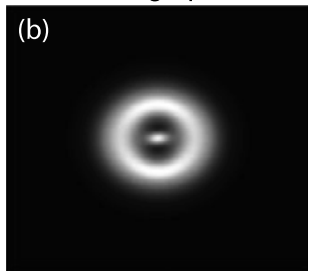

(e)

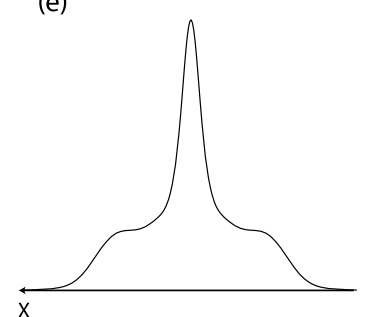

(h)

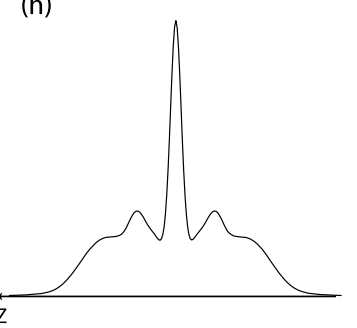

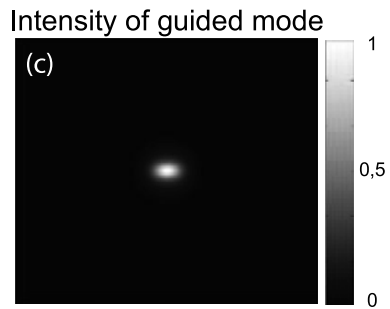
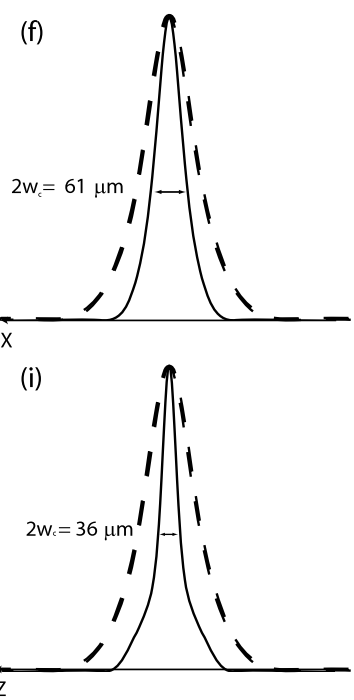

Figure 4. The index modulation distribution induced by a one-charge vortex beam after $t=1.35 \mathrm{~s}$ induction time (a), and the corresponding profiles along the $X(\mathrm{~d})$ and $Z(\mathrm{~g})$ axes. The intensity distribution at the exit face of a $9 \mathrm{~mm}$ long guiding structure when probed with a large beam ((b), (e), (h)) and when probed with a focused beam ((c), (f), (i)). The dashed curves show the beam profile without the guiding structure.

index modulation along the $Z$ axis (figures $4(\mathrm{~g})-(\mathrm{i})$ ) than along the $X$ axis (figures $4(\mathrm{~d})-(\mathrm{f})$ ).

\section{Experimental study}

To verify these numerical predictions, experimental studies are performed. A single topological charge vortex $(m=1)$ is imprinted on a $473 \mathrm{~nm} \mathrm{CW}$ laser using a reflective hologram fabricated by photolithography on a silicon wafer. Experiments are realized with a typical power of $100 \mu \mathrm{W}$ and the vortex beam is focused before the crystal with a $200 \mathrm{~mm}$ focal length to form an external diameter beam of about $120 \mu \mathrm{m}$ at the crystal input. The sample is cut from a $\mathrm{LiNbO}_{3}$ wafer doped with $0.01 \%$ of iron to increase the photorefractive effect. The beam is polarized along the ordinary axis of the crystal and propagates along a $9 \mathrm{~mm}$ long distance perpendicular to the crystal $c$-axis. 


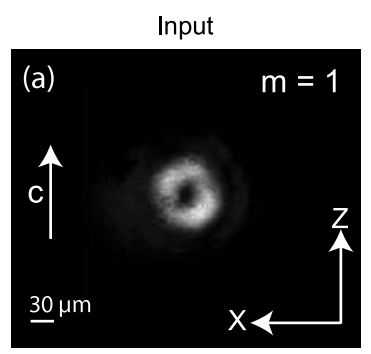

(c)
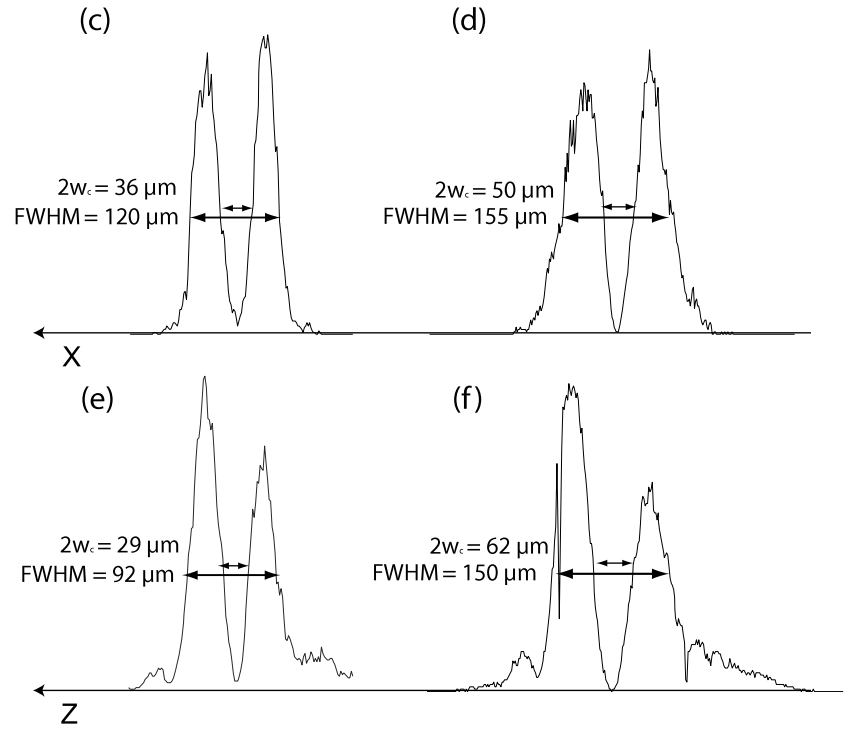

Figure 5. Experimental images at the $\mathrm{LiNbO}_{3}: \mathrm{Fe}$ crystal input (a) and output (b) and corresponding profiles along the $X((\mathrm{c}),(\mathrm{d}))$ and $Z$ ((e), (f)) axes.
The input and output faces of the crystal are imaged on a CCD camera. Figures 5(a) and (b) show the images of a one-charged vortex at the input and the output of the crystal, respectively, before the photovoltaic-photorefractive effect occurs. The respective profiles along the $X($ (c) and (d)) and $Z$ ((e) and (f)) axes are also presented. The vortex singularity shows a slightly elliptical dark core, whose dimensions are $36 \mu \mathrm{m}$ along the $X$ and $29 \mu \mathrm{m}$ along the $Z$ at the input and respectively 50 and $62 \mu \mathrm{m}$ at the output (figure 5(b)) in the linear regime. The beam intensity distribution is thus similar to the ones considered for numerical calculations. The ellipticity can be explained by astigmatism in the experimental setup.

The evolution of the output vortex beam is then monitored as the photorefractive defocusing effect is taking place. The dynamic presented in figure 6 lasts about 50 min before beam dislocation occurs. We observe that light gradually spreads out while the dark core slowly shrinks. Light goes away from the lower index region to concentrate in higher index areas on both sides. Moreover, the nonlinear effect combined with the rotating phase of the vortex breaks the vertical and horizontal symmetries. As a consequence the light seems to rotate anticlockwise (figure 6(d)). These experimental results are in good agreement with the numerical predictions.

However, discrepancies between the numerical calculations and experiments exist. In particular, a large discrepancy is evident for the time-scale to observe the phenomena in the experiments. The response is about two orders of magnitude slower experimentally than in the simulations. The main reason is the very partial knowledge of the photoexcitation cross section at the wavelength of interest. Moreover, many other crystal parameters, that strongly influence the response
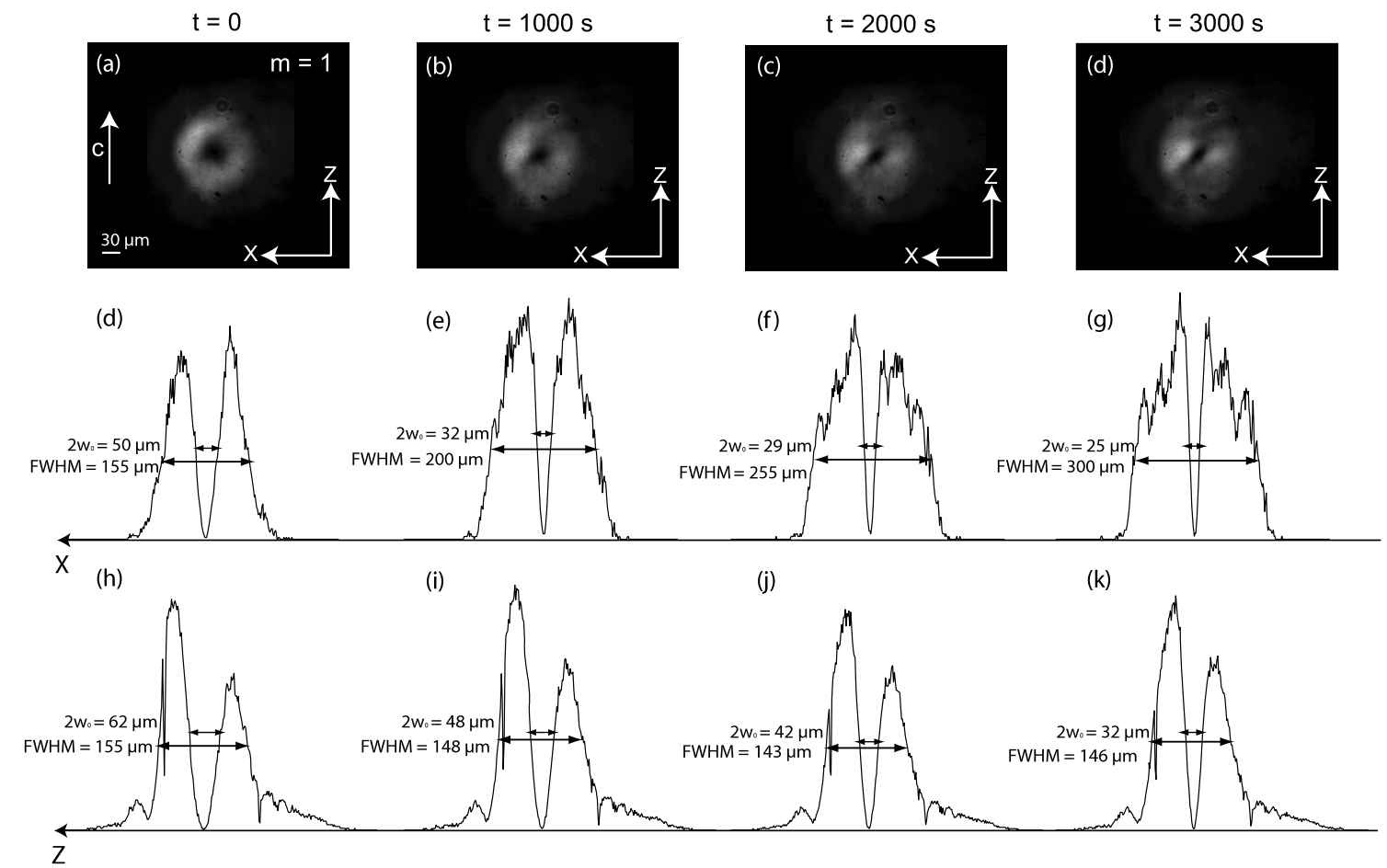

Figure 6. Experimental intensity distribution evolution at the exit face ((a)-(d)) for different induction times and corresponding profiles along the $X((\mathrm{~d})-(\mathrm{g}))$ and $Z((\mathrm{~h})-(\mathrm{k}))$ axes. 

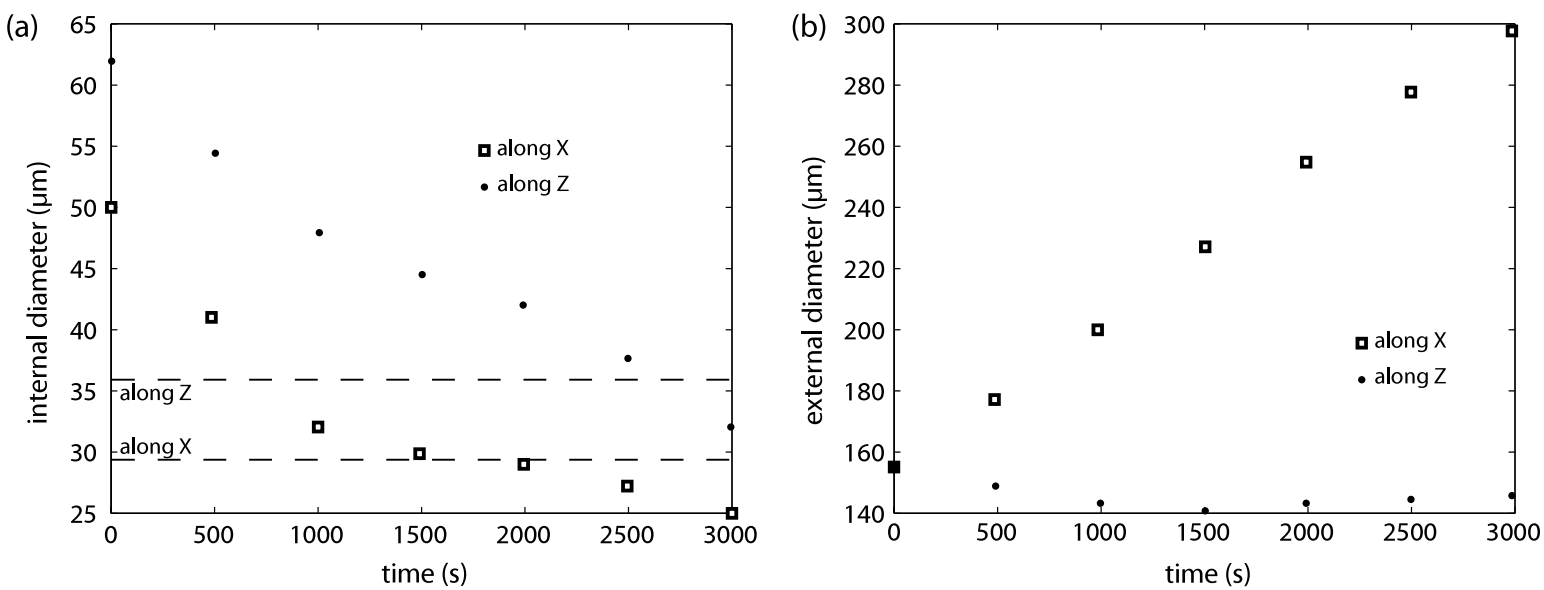

Figure 7. Temporal vortex size dimension evolution at the crystal output: internal (a) and external (b) FWHM along the $X$ (open squares) and $Z$ (filled circles) axes. The input internal diameter is represented by the dashed lines.

time of the photorefractive effect, such as the active iron concentration, have been set to arbitrary values. Adjustment of parameters to obtain similar response times between the model and the experiments was not pursued.

In figure 7 , experimental points showing the temporal evolution of the vortex characteristic dimensions are presented. In figure 7(a) we observe that the core is efficiently selftrapped along both transverse axes as expected from numerical simulation. In addition the initial beam core ellipticity vanishes and tends to become more circular. Finally, the vortex core maintains an almost constant size of about $30 \mu \mathrm{m}$ over propagation. Note also that the external beam diameter (figure 7(b)) enlarges more rapidly along the $X$ axis than along the $Z$ axis in accordance with the predictions from figure 3(b). We, however, observe that the beam diameter along the polar axis $Z$ is unexpectedly unaffected; this may be due to the initial elliptical vortex core. Note that in these experiments focusing dynamics up to the quasi-steady-state regime are considered. As a consequence the refractive index change does not reach saturation. In figure 7(a) we observe that the core width tends to reach a minimum value which is the sign of the approaching quasi-steady-state regime; a longer experiment gives beam dislocation.

The holographic storage time in $\mathrm{LiNbO}_{3}$ can extend up to several months. As a consequence, the index structure written by the vortex beam can be subsequently probed to check its properties. In the present work, the probe beam is a strongly attenuated $473 \mathrm{~nm}$ beam unable to alter the memorized structure. To check the overall index modulation, the crystal is first illuminated by a large weakly diffracted beam. The wave propagation is perturbed by the written index modulation present along the crystal. At the exit face the beam distribution gives a qualitative shape (figure $8(\mathrm{a})$ ) very similar to the calculated index modulation $\Delta n$ (figure 4(a)) where brighter areas correspond to higher index changes. In a second step a $25 \mu \mathrm{m}$ diameter low power Gaussian beam is injected in the central part of the induced structure. At the output, a confined spot is observed witnessing the presence of a 2D waveguide (figure 8(b)). Mode FWHM $36 \mu \mathrm{m}$ along the
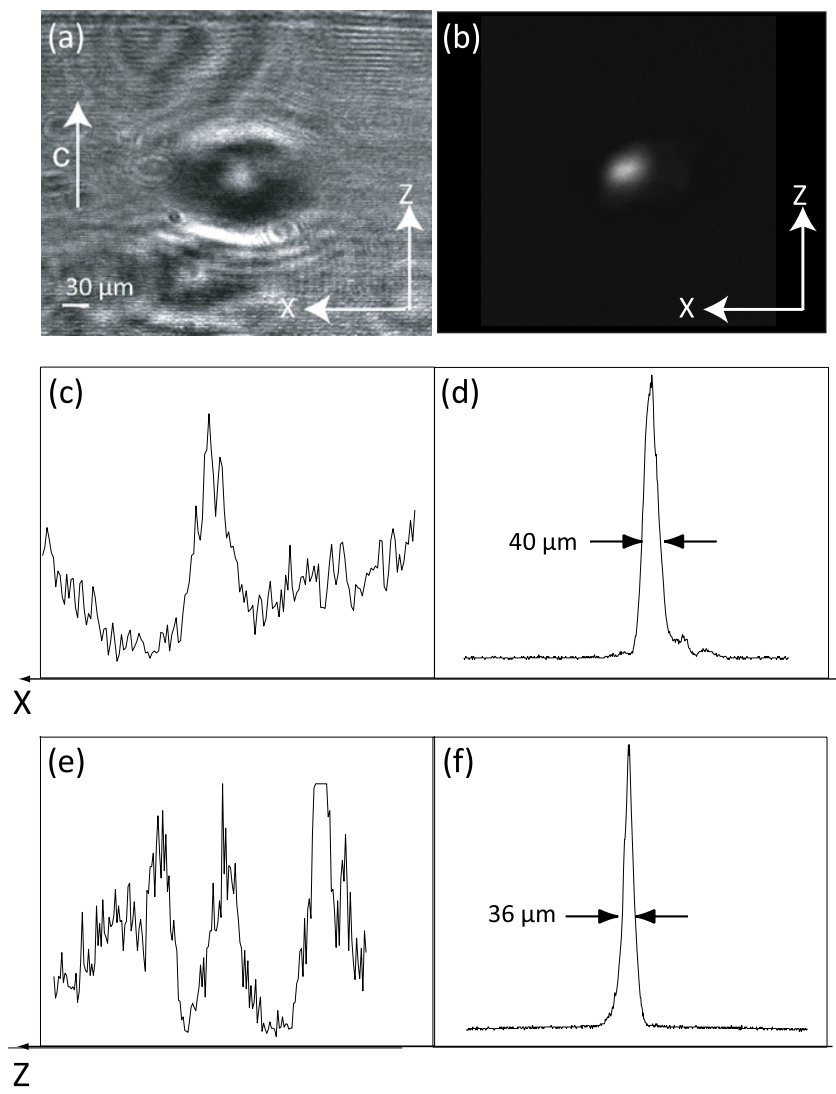

Figure 8. Intensity distribution at the output face of the $\mathrm{LiNbO}_{3}$ crystal inscribed with a vortex written index structure when probed with a large input beam (a) and when probed with a beam focused in the core (b). Corresponding profiles along the $X((\mathrm{c}),(\mathrm{d}))$ and $Z((\mathrm{e})$, (f)) axes.

$Z$ axis (figure 8(d)) and $41 \mu \mathrm{m}$ along the $X$ axis (figure $8(\mathrm{f})$ ) is confirmed by the numerical results (figure 4 ).

\section{Conclusion}

We showed that finite size vortex beams in a defocusing photovoltaic medium such as $\mathrm{LiNbO}_{3}$ tend to dislocate due to 
the anisotropy of the nonlinear effect. However, vortex selftrapping in the transient regime can inscribe $2 \mathrm{D}$ waveguides in the medium in place of the vortex dark core. Formation of the guiding structures is analyzed experimentally and with the help of a numerical model to reveal a complex refractive index distribution. Finite size vortices are shown to be viable tools to photo-induce waveguides. Sequential writing with annular vortex beams can authorize the formation of multiple guiding structures inside bulk $\mathrm{LiNbO}_{3}$.

\section{References}

[1] Shimura T, Matoba O, Inujima T and Kuroda K 1998 Segmented photorefractive waveguides in $\mathrm{LiNbO}_{3}: \mathrm{Fe} J$. Opt. Soc. Am. B 15 2006-12

[2] Zhang P, Zhao J, Yang D, Li B, Yang D and Feng X 2003 Optical masks prepared by using a liquid-crystal light valve for light-induced photorefractive waveguides Appl. Opt. 42 4208-11

[3] Zhang P, Yang D, Zhao J and Wang M 2006 Photo-written waveguides in iron-doped lithium niobate crystal employing binary optical masks Opt. Eng. 45074603

[4] Zhang P, Ma Y, Zhao J, Yang D and Xu H 2006 One-dimensional spatial dark soliton-induced channel waveguides in lithium niobate crystal Appl. Opt. 45 2273-8

[5] Stegeman G I A, Christodoulides D N and Segev M 2000 Optical spatial solitons: historical perspectives IEEE J. Quantum Electron. 6 1419-27

[6] Hasegawa A and Tappert F 1973 Transmission of stationary nonlinear optical pulses in dispersive dielectric fibers: I. Anomalous dispersion Appl. Phys. Lett. 23 142-4

[7] Barthélémy A, Maneuf S and Froehly C 1985 Soliton propagation and self-trapping of laser beams by a Kerr optical nonlinearity Opt. Commun. 55 201-6

[8] Assanto G and Stegeman G 2002 Simple physics of quadratic spatial solitons Opt. Express 10 388-96

[9] Eisenberg H S, Morandotti M and Silberberg Y 2001 Kerr spatiotemporal self-focusing in a planar glass waveguide Phys. Rev. Lett. 87 3902-5

[10] Chauvet M, Hawkins S A, Salamo G, Segev M, Bliss D F and Bryant G 1996 Self-trapping of planar optical beams by use of the photorefractive effect in InP:Fe Opt. Lett. 21 1333-5

[11] Lam L and Prost J 1992 Solitons in Liquid Crystals (New York: Springer/Verlag)

[12] Segev M, Crosignani B, Yariv A and Fischer B 1992 Spatial solitons in photorefractive media Phys. Rev. Lett. 68 923-6

[13] Duree G, Shultz G J, Salamo J L, Segev M, Yariv A, Crosignani B, Di Porto P, Sharp E J and Neurgaonkar R R 1993 Observation of self-trapping of an optical beam due to the photorefractive effect Phys. Rev. Lett. 71 533-6

[14] Singh S R and Christodoulides D N 1995 Evolution of spatial optical solitons in biased photorefractive media under steady-state conditions Opt. Commun. 118 569-76

[15] Duree G, Moris M and Salamo G 1995 Dark photorefractive spatial solitons and photorefractive vortex solitons Phys. Rev. Lett. 74 1978-81

[16] Segev M, Valley G C, Bashaw M C, Taya M, Fejer M M and Ginzton E L 1997 Photovoltaic solitons J. Opt. Soc. Am. B $141772-81$
[17] Fressengeas N, Maufoy J and Kugel G 1996 Temporal behaviour of bidimensional photorefractive bright spatial solitons Phys. Rev. E 54 6866-75

[18] Mamaev A V, Saffman M and Zozulya A A 1997 Time-dependent evolution of an optical vortex in photorefractive media Phys. Rev. A 56 1713-6

[19] Chauvet M 2003 Temporal analysis of open-circuit dark photovoltaic spatial solitons J. Opt. Soc. Am. B 20 2515-22

[20] Chen Z, Mitchell M and Segev M 1996 Steady-state photorefractive soliton-induced y-junction waveguides and high-order dark spatial solitons Opt. Lett. 21 716-8

[21] Mamaev A V, Saffman M and Zozulya A A 1997 Decay of high order optical vortices in anisotropic nonlinear optical media Phys. Rev. Lett. 78 2108-11

[22] Fazio E, Renzi R, Rinaldi F, Bertolotti M, Chauvet M, Ramadan W, Petris A and Vlad V I 2004 Screeningphotovoltaic bright solitons in lithium niobate and associated single-mode waveguides Appl. Phys. Lett. 852193

[23] Taya M, Bashaw M C, Fejer M M, Segev M and Valley G C 1995 Observation of dark photovoltaic spatial solitons Phys. Rev. A 52 3095-100

[24] She W L, Lee K K and Lee W K 1999 Observation of two-dimensional bright photovoltaic spatial solitons Phys. Rev. Lett. 83 3182-5

[25] Curtis J E and Grier D 2003 Modulated optical vortices Opt. Lett. 28 872-4

[26] Madison K W, Chevy F, Wohlleben W and Dalibard J 2000 Vortex formation in a stirred Bose-Einstein condensates Phys. Rev. Lett. 84 806-8

[27] Mamaev A V, Saffman M and Zozulya A A 1996 Propagation of dark stripe beams in nonlinear media: snake instability and creation of optical vortices Phys. Rev. Lett. 76 2262-5

[28] Gan X, Zhang P, Liu S, Zheng Y, Zhao J and Chen Z 2009 Stabilization and breakup of optical vortices in presence of hybrid nonlinearity Opt. Express 1723130

[29] Chen Z, Feng Shih M, Segev M, Wilson D W, Muller R E and Maker P D

1997 Steady-state vortex-screening solitons formed in biased photorefractive media Opt. Lett. 22 1751-3

[30] Chen Z, Segev M, Wilson D W, Muller R E and Maker P D 1997 Self-trapping of an optical vortex by use of the bulk photovolaic effect Phys. Rev. Lett. 78 2948-51

[31] Devaux F, Coda-Bouchot V, Chauvet M and Passier R 2008 New time-dependent photorefractive 3D model: application to self-trapped beam with large bending J. Opt. Soc. Am. B 25 1081-6

[32] Kukhtarev N, Markov V B, Odulov S G, Soskin M S and Vinetskii V L 1979 Holographic storage in electrooptic crystals. I. Steady state Ferroelectrics 22949

[33] Passier R, Devaux F and Chauvet M 2008 Impact of tensorial nature of the electro-optic effect on vortex beam propagation in photorefractive media Opt. Express 16 7134-41

[34] Basistiy I V, Soskin M S and Vasnetsov M V 1995 Optical wavefront dislocations and their properties Opt. Commun. $119604-12$

[35] Simon M, Wevering S, Buse K and Krätzig E 1997 The bulk photovoltaic effect of photorefractive $\mathrm{LiNbO}_{3}: \mathrm{Fe}$ crystals at high light intensities J. Phys. D: Appl. Phys. 30 144-9

[36] Yan W et al 2006 Influence of composition on the photorefractive centers in pure $\mathrm{LiNbO}_{3}$ at low light intensity Appl. Opt. 45 2453-8 